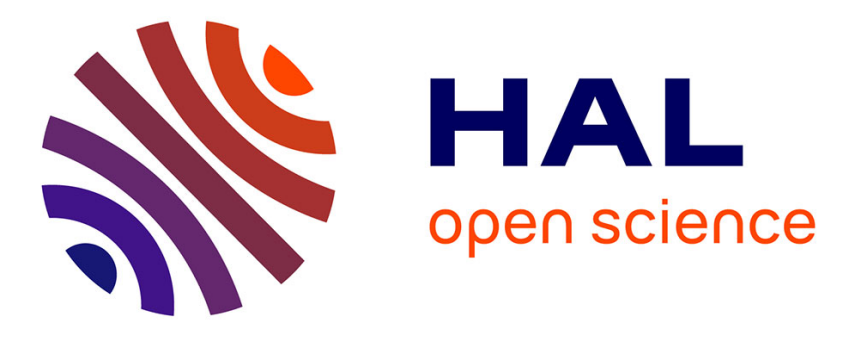

\title{
An equivalent representation of the Brown-Resnick process
}

\author{
S. Engelke, Z. Kabluchko, M. Schlather
}

\section{To cite this version:}

S. Engelke, Z. Kabluchko, M. Schlather. An equivalent representation of the Brown-Resnick process. Statistics and Probability Letters, 2011, 10.1016/j.spl.2011.03.010 . hal-00753951

\section{HAL Id: hal-00753951 \\ https://hal.science/hal-00753951}

Submitted on 20 Nov 2012

HAL is a multi-disciplinary open access archive for the deposit and dissemination of scientific research documents, whether they are published or not. The documents may come from teaching and research institutions in France or abroad, or from public or private research centers.
L'archive ouverte pluridisciplinaire HAL, est destinée au dépôt et à la diffusion de documents scientifiques de niveau recherche, publiés ou non, émanant des établissements d'enseignement et de recherche français ou étrangers, des laboratoires publics ou privés. 


\section{Accepted Manuscript}

An equivalent representation of the Brown-Resnick process

S. Engelke, Z. Kabluchko, M. Schlather

PII:

S0167-7152(11)00093-9

DOI:

10.1016/j.spl.2011.03.010

Reference: $\quad$ STAPRO 5945

To appear in: $\quad$ Statistics and Probability Letters

Received date: 5 October 2010

Revised date: 1 March 2011

Accepted date: 3 March 2011

Please cite this article as: Engelke, S., Kabluchko, Z., Schlather, M., An equivalent representation of the Brown-Resnick process. Statistics and Probability Letters (2011), doi:10.1016/j.spl.2011.03.010

This is a PDF file of an unedited manuscript that has been accepted for publication. As a service to our customers we are providing this early version of the manuscript. The manuscript will undergo copyediting, typesetting, and review of the resulting proof before it is published in its final form. Please note that during the production process errors may be discovered which could affect the content, and all legal disclaimers that apply to the journal pertain. 


\title{
An equivalent representation of the Brown-Resnick process
}

\author{
S. Engelke ${ }^{\mathrm{a}, *}$, Z. Kabluchko ${ }^{\mathrm{b}}$, M. Schlather ${ }^{\mathrm{a}}$ \\ ${ }^{a}$ Insitut für Mathematische Stochastik, Georg-August-Universität Göttingen, Goldschmidtstr. 7, D-37077 Göttingen, Germany \\ ${ }^{b}$ Institut für Stochastik, Universität Ulm, Helmholtzstr. 18, D-89069 Ulm, Germany
}

\begin{abstract}
Brown and Resnick (1977) introduce a max-stable process that is obtained as a limit of maxima of independent Ornstein-Uhlenbeck processes. As shown in Kabluchko et al. (2009) this process is dissipative and it therefore admits a mixed moving maxima representation. We show that the distribution of the spectral functions in this representation equals a well-known diffusion, namely a standard Brownian motion with drift conditional on taking negative values only. This can be used for fast simulation methods.
\end{abstract}

Keywords: Brown-Resnick process, mixed moving maxima, dissipative, conditional negative Brownian motion, simulation of max-stable processes

\section{Introduction}

The Brown-Resnick process was first introduced in Brown and Resnick (1977) as the stochastic process given by

$$
\Psi(t)=\bigvee_{i=1}^{\infty}\left[X_{i}+B_{i}(t)\right], t \in \mathbb{R},
$$

where $B_{i}, i \in \mathbb{N}$, are independent copies of a standard Brownian motion on the real line with drift $-|t| / 2$ and $\sum_{i \in \mathbb{N}} \delta_{X_{i}}$ is a Poisson point process on the real axis with intensity $e^{-x} \mathrm{~d} x, x \in \mathbb{R}$, independent of the $B_{i}, i \in \mathbb{N}$. Moreover, it was shown that $\Psi$ is stationary. This property still holds if the Brownian motion is replaced by any Gaussian process with stationary increments and a suitable drift (Kabluchko et al., 2009). The major importance of these processes arise from the fact that they constitute the limits of maxima of suitably normalized and rescaled Gaussian processes. This makes them an interesting objective in applications such as modeling extreme events (Buishand et al., 2008). To this end, it is crucial to obtain exact and efficient simulation methods. However, algorithms based on the standard definition of Brown-Resnick processes as in (1) yield very slow convergence. Alternative simulation techniques have already been considered in Oesting et al. (2011) which are also applicable to the generalization of $\Psi$ by Kabluchko et al. (2009). In the situation of the process in (1) the Markovian structure of Brownian motion can beneficially be used to establish a mixed moving maxima representation of $\Psi$.

\footnotetext{
${ }^{*}$ Corresponding author

Email addresses: sengelk@uni-goettingen.de (S. Engelke), zakhar.kabluchko@uni-ulm.de (Z. Kabluchko), schlather@math.uni-goettingen.de (M. Schlather)
} 
Definition 1. Let $\{F(t)\}_{t \in \mathbb{R}}$ be a measurable process and suppose that $\mathbb{E} \int_{\mathbb{R}} \exp (F(t)) \mathrm{d} t<\infty$. Further, let $\sum_{i \in \mathbb{N}} \delta_{\left(t_{i}, y_{i}\right)}$ be a Poisson point process on $\mathbb{R} \times \mathbb{R}$ with intensity $\mathrm{d} t e^{-y} \mathrm{~d} y$ and let $F_{i}, i \in \mathbb{N}$, be independent copies of $F$. A process of the form

$$
\zeta(t)=\bigvee_{i=1}^{\infty}\left[y_{i}+F_{i}\left(t-t_{i}\right)\right], t \in \mathbb{R},
$$

is called a mixed moving maxima process.

It was shown (Theorem 14 in Kabluchko et al. (2009)) that $\Psi$ admits a representation as a mixed moving maxima process (i.e., it is a dissipative process, cf. Wang and Stoev (2010)). The distribution of the process $F$ in Definition 1 is however still unknown. In this paper we show that this distribution equals a well known diffusion process. Namely, it is a standard Brownian motion with drift $-|t| / 2$ conditioned on not taking positive values.

\section{Theorem and proof}

Theorem 1. Let $B$ be a Brownian motion on the real line with drift $-|t| / 2$ and $B(0)=0$. Then the following two processes have the same distribution:

1.

$$
\Psi_{1}(t)=\bigvee_{i=1}^{\infty}\left[X_{i}+B_{i}(t)\right], t \in \mathbb{R},
$$

where $B_{i}, i \in \mathbb{N}$, are independent copies of $B$ and $\sum_{i \in \mathbb{N}} \delta_{X_{i}}$ is a Poisson point process on the real axis with intensity $e^{-x} \mathrm{~d} x, x \in \mathbb{R}$, independent of the $B_{i}, i \in \mathbb{N}$.

2.

$$
\Psi_{2}(t)=\bigvee_{i=1}^{\infty}\left[V_{i}+R_{i}\left(t-S_{i}\right)\right], t \in \mathbb{R},
$$

where $\sum_{i \in \mathbb{N}} \delta_{\left(S_{i}, V_{i}\right)}$ is a Poisson point process on $\mathbb{R} \times \mathbb{R}$ with intensity $\frac{1}{2} \mathrm{~d} t e^{-x} \mathrm{~d} x, t, x \in \mathbb{R}$, independent of the $R_{i}, i \in \mathbb{N}$. The $R_{i}$ 's are independent copies of the process

$$
R(t)=1_{t \leq 0} R_{-}(-t)+1_{t \geq 0} R_{+}(t),
$$

where $R_{-}$and $R_{+}$are independent processes which follow $t \geq 0$ the law of the nonpositive diffusion determined by the stochastic differential equation for

$$
\mathrm{d} \tilde{R}(t)=\mathrm{d} W(t)+\frac{1}{2} \operatorname{coth}(\tilde{R}(t) / 2) \mathrm{d} t,
$$

starting at $R_{+}(0)=R_{-}(0)=0$, where $W$ is a standard Brownian motion.

Remark 1. For $y \leq 0$, the diffusion defined by (4) and started at $\tilde{R}(0)=y$ has the same distribution as a Brownian motion $\{B(t)\}_{t \geq 0}$ with drift $-t / 2$ and $B(0)=y$ conditioned on not taking positive values for all $t \geq 0$. For details see Lemma 55.1 in Rogers and Williams (2000).

In fact, for $y=0$, it follows from Theorem 3 in Rogers and Pitman (1981) that the opposite of the diffusion, namely $-\tilde{R}$, has the same distribution as a three dimensional Bessel process of drifting Brownian motion. More precisely, it can be obtained by taking the radial part of a Brownian motion in $\mathbb{R}^{3}$ with drift $1 / 2$ in the direction of the first coordinate axis. This is particularly important with regard to efficient simulation methods. 
Remark 2. In fact, we will prove more than the equality in law of $\Psi_{1}$ and $\Psi_{2}$. We will show that the random families of functions $\left\{X_{i}+B_{i}(\cdot)\right\}_{i \in \mathbb{N}}$ and $\left\{V_{i}+R_{i}\left(\cdot-S_{i}\right)\right\}_{i \in \mathbb{N}}$ considered as point processes on $C(\mathbb{R})$, the space of continuous functions on $\mathbb{R}$, have the same law.

Proof of Theorem 1. Let us first set up some notation. For the Brownian motion $B$ with drift $-|t| / 2$ and $B(0)=0$ let $Y=\sup _{s \in \mathbb{R}} B(s)$ and $T=\arg \sup _{s \in \mathbb{R}} B(s)$. Moreover, denote by $Z(t)=B(t+T)-Y$ the process $B$ whose supremum is shifted into the origin.

Consider the Poisson point process $\sum_{i \in \mathbb{N}} \delta_{\left(X_{i}, B_{i}\right)}$ on $\mathbb{R} \times C(\mathbb{R})$ with intensity $e^{-x} \mathrm{~d} x \mathbb{P}_{B}(\mathrm{~d} f)$, where $C(\mathbb{R})$ denotes the space of continuous functions on $\mathbb{R}$ and $\mathbb{P}_{B}$ is the law of $B$. The mapping

$$
\eta: \mathbb{R} \times C(\mathbb{R}) \rightarrow C(\mathbb{R}) \times \mathbb{R} \times \mathbb{R},(u, f) \mapsto(f((\cdot)+\arg \sup f)-\sup f, \arg \sup f, \sup f+u)
$$

is measurable (see proof of Lemma 2.13 in Oesting et al. (2011) for details), where the arg sup is the infimum of all points where the supremum is attained. The key idea of the proof is to compute the intensity measure $\Phi$ of the Poisson point process on $C(\mathbb{R}) \times \mathbb{R} \times \mathbb{R}$ induced by $\eta$, namely

$$
\sum_{i \in \mathbb{N}} \delta_{\left(B_{i}\left((\cdot)+\arg \sup B_{i}\right)-\sup B_{i}, \arg \sup B_{i}, \sup B_{i}+X_{i}\right)} .
$$

The main difficulty here is to find the distribution of the random element $(Z, T, Y)$ which is essential in the later calculations. More precisely, for $t_{1} \leq \ldots \leq t_{n} \in \mathbb{R}, y_{1}, \ldots, y_{n}<0, n \in \mathbb{N}, t \in \mathbb{R}$ and $y \in \mathbb{R}_{+}$we will later establish the equation

$$
\mathrm{d} \mathbb{P}\left(Z\left(t_{1}\right)=y_{1} \ldots, Z\left(t_{n}\right)=y_{n}, T=t, Y=y\right)=\mathrm{d} \mathbb{P}\left(R\left(t_{1}\right)=y_{1}, \ldots, R\left(t_{n}\right)=y_{n}, R(-t)=-y\right) \frac{e^{-y}}{2},
$$

where $R$ is the two-sided diffusion process which satisfies (4) and starts in 0 . Thus, the distribution of the shifted Brownian motion $Z$ essentially equals the one of the reweighted diffusion $R$. In order to compute the measure $\Phi$ let $M \times I \times D \in \mathcal{B}(C(\mathbb{R})) \otimes \mathcal{B}(\mathbb{R}) \otimes \mathcal{B}(\mathbb{R})$ be a measurable set with $M=C_{t_{1}, \ldots, t_{n}}(E)=\{g \in C(\mathbb{R})$ : $\left.\left(g\left(t_{1}\right), \ldots, g\left(t_{n}\right)\right) \in E\right\}$, where $E \in \mathcal{B}\left(\mathbb{R}^{n}\right)$. Furthermore, denote by $(\Omega, \mathcal{A}, \mathbb{P})$ the underlying probability space.

$$
\begin{aligned}
\Phi(M \times I \times D) & =\int_{\mathbb{R}} e^{-x} \mathbb{P}((Z, \arg \sup B, \sup B) \in M \times I \times D-(0,0, x)) \mathrm{d} x \\
& =\int_{\mathbb{R}} e^{-x} \mathbb{P}\left(\left(Z\left(t_{1}\right), \ldots, Z\left(t_{n}\right)\right) \in E, \arg \sup B \in I, \sup B \in D-x\right) \mathrm{d} x \\
& =\int_{\mathbb{R}} e^{-x} \int_{I} \int_{D} \int_{E} \mathbb{P}\left(Z\left(t_{1}\right)=y_{1}, \ldots, Z\left(t_{n}\right)=y_{n}, \arg \sup B=t, \sup B=y-x\right) \\
& =\int_{\mathbb{R}} e^{-x} \int_{I} \int_{D} \int_{E} \mathbb{P}\left(R\left(t_{1}\right)=y_{1}, \ldots, R\left(t_{n}\right)=y_{n}, R(-t)=-(y-x)\right) \frac{1}{2} e^{-(y-x)} \mathrm{d} y \mathrm{~d} t \mathrm{~d} x \\
& =\int_{D} \frac{1}{2} e^{-y} \int_{I} \int_{E} \int_{\mathbb{R}} \mathbb{P}\left(y_{1}, \ldots, y_{n}\right) \mathrm{d} y \mathrm{~d} t \mathrm{~d} x \\
& =\int_{D} \frac{1}{2} e^{-y} \int_{I} \int_{E} \mathbb{P}\left(R\left(t_{1}\right)=y_{1}, \ldots, R\left(t_{n}\right)=y_{n}, R(-t)=-(y-x)\right) \\
& =\mathbb{P}_{R}\left(C_{t_{1}, \ldots, t_{n}}(E)\right) \int_{I} \frac{1}{2} \mathrm{~d} t \int_{D} e^{-y} \mathrm{~d} y, R\left(y_{1}, \ldots, y_{n}\right) \mathrm{d} t \mathrm{~d} y
\end{aligned}
$$


where the fourth equality follows from (6) and $\mathbb{P}_{R}$ is the law of $R$ on $C(\mathbb{R})$.

Therefore, the intensity $\Phi$ of the Poisson point process (5) is given by $\mathbb{P}_{R}(\mathrm{~d} f) \frac{1}{2} \mathrm{~d} t e^{-x} \mathrm{~d} x$. On the other hand, if we consider a Poisson point process $\sum_{i \in \mathbb{N}} \delta_{\left(S_{i}, V_{i}\right)}$ with intensity $\frac{1}{2} \mathrm{~d} t e^{-x} \mathrm{~d} x$ and independent marks $R_{i} \sim \mathbb{P}_{R}$, $i \in \mathbb{N}$, then we also obtain a Poisson point process $\sum_{i \in \mathbb{N}} \delta_{\left(R_{i}, S_{i}, V_{i}\right)}$ with intensity $\mathbb{P}_{R}(\mathrm{~d} f) \frac{1}{2} \mathrm{~d} t e^{-x} \mathrm{~d} x$. This implies the equality in distribution of (2) and (3).

Let us now proceed with the proof of equation (6). To this end, we will first relate the distribution of $(T, Y)$ to the one-dimensional distributions of the diffusion $R$. In a second step, we then compute the distribution of the shifted Brownian motion $Z$ conditional on $(T, Y)$.

By formulae 2.1.1.4 and 2.1.13.4 in Borodin and Salminen (1996) it follows that the bivariate random variable $(T, Y)$ has the density

$$
\mathrm{d} \mathbb{P}(T=t, Y=y)=f(|t|, y)\left(1-e^{-y}\right) \mathrm{d} t \mathrm{~d} y, \quad t \in \mathbb{R}, y \in \mathbb{R}_{+},
$$

where $f$ is given by

$$
f(t, y)=\frac{y}{\sqrt{2 \pi} t^{3 / 2}} \exp \left(-\frac{(y+t / 2)^{2}}{2 t}\right)=-e^{-y} f(t,-y) .
$$

Note that $\left(1-e^{-y}\right)$ is the probability that $\{B(s): s \geq 0\}$ never hits $y \in \mathbb{R}_{+}$. On the other hand, we can explicitly compute the entrance law and the transition densities of the diffusion $\{R(t)\}_{t \geq 0}$ satisfying (4) and started at $R(0)=0$. For $x, y<0$ let

$$
p_{t}(x, y) \mathrm{d} y=\mathrm{d} \mathbb{P}\left(\sup _{0 \leq s \leq t} B(s) \leq 0, B(t)=y \mid B(0)=x\right), \quad t>0 .
$$

By Lemma 55.1 in Rogers and Williams (2000) we conclude that

$$
p_{t}(x, y)=(2 \pi t)^{-1 / 2} e^{-(y-x) / 2-t / 8}\left[e^{-(y-x)^{2} /(2 t)}-e^{-(y+x)^{2} /(2 t)}\right]
$$

and

$$
p_{t}(x, y)=e^{-(y-x)} p_{t}(y, x)
$$

Thus, formula 2.1.1.4(1) in Borodin and Salminen (1996) shows that the transition density of $R$ has the form

$$
q_{t}(x, y) \mathrm{d} y=\mathrm{d} \mathbb{P}\left(B(t)=y \mid B(0)=x, \sup _{0 \leq s \leq \infty} B(s) \leq 0\right)=\left(1-e^{x}\right)^{-1} p_{t}(x, y)\left(1-e^{y}\right) \mathrm{d} y, \quad t>0, x, y<0 .
$$

Consequently, the entrance law $q_{t}(0, \cdot)$ of $R$, i.e., $q_{t}(0, y) \mathrm{d} y=\mathrm{d} \mathbb{P}(R(t)=y)$ for $t>0$ and $y<0$ is given by

$$
\begin{aligned}
q_{t}(0, y) & =\lim _{x \rightarrow 0} q_{t}(x, y)=\frac{1}{\sqrt{2 \pi t}} e^{-y / 2-t / 8}\left(1-e^{y}\right) \lim _{x \rightarrow 0} \frac{e^{-(y-x)^{2} /(2 t)}-e^{-(y+x)^{2} /(2 t)}}{1-e^{x}} \\
& =\frac{-2 y}{\sqrt{2 \pi} t^{3 / 2}} e^{-(y+t / 2)^{2} /(2 t)}\left(1-e^{y}\right) \\
& =-2 f(t, y)\left(1-e^{y}\right) .
\end{aligned}
$$

Note that this together with (7) implies

$$
\begin{aligned}
\mathrm{d} \mathbb{P}(T=t, Y=y) & =f(|t|, y)\left(1-e^{-y}\right) \mathrm{d} t \mathrm{~d} y \\
& =-f(|t|,-y)\left(1-e^{-y}\right) e^{-y} \mathrm{~d} t \mathrm{~d} y=\mathrm{d} \mathbb{P}(R(-t)=-y) \frac{e^{-y}}{2}, \quad t \in \mathbb{R}, y \in \mathbb{R}_{+} .
\end{aligned}
$$


We now look more closely at the distribution of $Z$ conditional on the maximum $(T, Y)$. To this end, without loss of generality, let $D_{t, y}=\{T=t, Y=y\}$ with $t, y \geq 0$. By Williams' path decomposition of Brownian motion (Theorem 55.9 in Rogers and Williams (2000)), $\left\{Z(s):-t \leq s \leq 0 \mid D_{t, y}\right\}$ is independent of $\left\{Z(s): s>0 \mid D_{t, y}\right\}$ and the latter has the same distribution as $\{R(s): s>0 \mid R(0)=0\}$, where $R$ satisfies (4). Furthermore, by the construction of $Z$ we have that the process $\left\{Z(s): s \leq-t \mid D_{t, y}\right\}$ is independent of $\left\{Z(s): s>-t \mid D_{t, y}\right\}$.

For $p, l, m \in \mathbb{N}$ and $r_{1} \leq \ldots \leq r_{p} \leq-t \leq s_{1} \leq \ldots \leq s_{l} \leq 0 \leq u_{1} \leq \ldots \leq u_{m}$ define random events

$$
\begin{aligned}
& A_{1}=\left\{Z\left(r_{1}\right)=w_{1}, \ldots, Z\left(r_{p}\right)=w_{p}\right\}, \\
& A_{2}=\left\{Z\left(s_{1}\right)=x_{1}, \ldots, Z\left(s_{l}\right)=x_{l}\right\}, \\
& A_{3}=\left\{Z\left(u_{1}\right)=z_{1}, \ldots, Z\left(u_{m}\right)=z_{m}\right\},
\end{aligned}
$$

where $w_{1}, \ldots, w_{p}, x_{1}, \ldots, x_{l}, z_{1}, \ldots, z_{m} \leq 0$. The above considerations yield

$$
\mathrm{d} \mathbb{P}\left(A_{1} \cap A_{2} \cap A_{3} \mid D_{t, y}\right)=\mathrm{d} \mathbb{P}\left(A_{1} \mid D_{t, y}\right) \mathrm{d} \mathbb{P}\left(A_{2} \mid D_{t, y}\right) \mathrm{d} \mathbb{P}\left(A_{3} \mid D_{t, y}\right) .
$$

As already mentioned above, we have

$$
\mathrm{d} \mathbb{P}\left(A_{3} \mid D_{t, y}\right)=\mathrm{d} \mathbb{P}\left(R\left(u_{1}\right)=z_{1}, \ldots, R\left(u_{m}\right)=z_{m} \mid R(0)=0\right) .
$$

Moreover,

$$
\begin{aligned}
\mathrm{d} \mathbb{P}\left(A_{1} \mid D_{t, y}\right) & =\mathrm{d} \mathbb{P}\left(B\left(r_{1}+t\right)=w_{1}+y, \ldots, B\left(r_{p}+t\right)=w_{p}+y \mid B(0)=0, B(s) \leq y \forall s \leq 0\right) \\
& =\mathrm{d} \mathbb{P}\left(B\left(r_{1}+t\right)=w_{1}, \ldots, B\left(r_{p}+t\right)=w_{p} \mid B(0)=-y, B(s) \leq 0 \forall s \leq 0\right) \\
& =\mathrm{d} \mathbb{P}\left(R\left(r_{1}+t\right)=w_{1}, \ldots, R\left(r_{p}+t\right)=w_{p} \mid R(0)=-y\right) \\
& =\mathrm{d} \mathbb{P}\left(R\left(r_{1}\right)=w_{1}, \ldots, R\left(r_{p}\right)=w_{p} \mid R(-t)=-y\right),
\end{aligned}
$$

where we used Remark 1 and the fact that $R$ is Markov.

For the second factor in (11) we first compute explicitly $\operatorname{dP}\left(A_{2} \cap D_{t, y}\right)$. To this end, put $s_{0}=-t, x_{0}=-y$, $\Delta s_{i}=s_{i}-s_{i-1}$ and $\xi=\left(x_{1}, \ldots, x_{l}, t, y\right)$.

$$
\begin{aligned}
\mathrm{d} \mathbb{P}\left(A_{2} \cap D_{t, y}\right) & =\mathrm{d} \mathbb{P}\left(Z\left(s_{1}\right)=x_{1}, \ldots, Z\left(s_{l}\right)=x_{l}, T=t, Y=y\right) \\
& =f\left(\left|s_{l}\right|,-x_{l}\right)\left(1-e^{x_{0}}\right) \prod_{i=1}^{l} p_{\Delta s_{i}}\left(x_{i-1}, x_{i}\right) \mathrm{d} \xi \\
& =f\left(\left|s_{l}\right|,-x_{l}\right)\left(1-e^{x_{0}}\right) e^{-\left(x_{l}-x_{0}\right)} \prod_{i=1}^{l} p_{\Delta s_{i}}\left(x_{i}, x_{i-1}\right) \mathrm{d} \xi \\
& =-f\left(\left|s_{l}\right|, x_{l}\right)\left(1-e^{x_{0}}\right) e^{x_{0}} \prod_{i=1}^{l} p_{\Delta s_{i}}\left(x_{i}, x_{i-1}\right) \mathrm{d} \xi \\
& =-f\left(\left|s_{l}\right|, x_{l}\right)\left(1-e^{x_{l}}\right) e^{x_{0}} \prod_{i=1}^{l} q_{\Delta s_{i}}\left(x_{i}, x_{i-1}\right) \mathrm{d} \xi \\
& =\frac{e^{-y}}{2} q_{\left|s_{l}\right|}\left(0, x_{l}\right) \prod_{i=1}^{l} q_{\Delta s_{i}}\left(x_{i}, x_{i-1}\right) \mathrm{d} \xi \\
& =\mathrm{d} \mathbb{P}\left(R\left(s_{1}\right)=x_{1}, \ldots, R\left(s_{l}\right)=x_{l}, R(-t)=-y\right) \frac{e^{-y}}{2},
\end{aligned}
$$


where we used (8) and (9). The second equation follows by an elementary computation of the transition probabilities for the shifted Brownian motion $Z$. Starting from the point $\left(s_{0}, x_{0}\right)$ it moves to $\left(s_{l}, x_{l}\right)$ and then attains the one-sided maximum at the origin (with probability $f\left(\left|s_{l}\right|,-x_{l}\right)$, see (7)). At the same time, the process $B$ stays below level $y$ on the negative half-axis, which has probability $\left(1-e^{x_{0}}\right)$. Thus, (10) yields

$$
\mathrm{d} \mathbb{P}\left(A_{2} \mid D_{t, y}\right)=\mathrm{d} \mathbb{P}\left(R\left(s_{1}\right)=x_{1}, \ldots, R\left(s_{l}\right)=x_{l} \mid R(-t)=-y\right) .
$$

Putting the parts together, formula (6) follows from (10) and (11).

\section{Simulation method}

The representation of the Brown-Resnick process in (3) turns out to be a promising basis for simulations. Schlather (2002) gives an algorithm for the simulation of mixed moving maxima processes where the spectral functions have finite support. Here, this approach can be adopted, the only difference being that in our case the spectral function, i.e., the conditional negative Brownian motion, does not have finite support. Diffusions from anywhere outside the simulation window can therefore influence the value of the Brown-Resnick process inside the simulation window. However, since the diffusions quickly tend to negative infinity, the probability of this event is exponentially decreasing with the size of the interval which is added on both sides of the simulation window. This error probability can therefore be made arbitrarily small. In fact, calculations show that, independent of the size of the simulation window, additional intervals of length 45 on both sides ensure that the probability of not drawing a path from the desired distribution is less than $0.5 \%$. Furthermore, paths starting in these additional intervals do not need to be simulated on the whole enlarged area since knowing the diffusion entrance law enables us to directly jump into the simulation window. Owing to these facts, the algorithm based on the mixed moving maxima representation is about 60 times faster than the more general algorithms considered in Oesting et al. (2011).

Another advantage of this simulation method is its flexibility. In particular, the technique is not restricted to grids but can easily be performed on any set of points. Moreover, also large simulation windows are feasible since the computational costs increase first quadratically, then linearly, for increasing simulation intervals.

\section{Acknowledgements}

The authors are grateful to Marco Oesting for helpful discussions and to an unknown referee for useful hints and comments. S. Engelke has been financially supported by Deutsche Telekom Stiftung.

\section{References}

Borodin, A. N., Salminen, P., 1996. Handbook of Brownian motion—facts and formulae. Probability and its Applications. Birkhäuser Verlag, Basel.

Brown, B. M., Resnick, S. I., 1977. Extreme values of independent stochastic processes. J. Appl. Probability $14,732-739$.

Buishand, T. A., de Haan, L., Zhou, C., 2008. On spatial extremes: with application to a rainfall problem. Ann. Appl. Stat. 2, 624-642. 
Kabluchko, Z., Schlather, M., de Haan, L., 2009. Stationary max-stable fields associated to negative definite functions. Ann. Probab. 37, 2042-2065.

Oesting, M., Kabluchko, Z., Schlather, M., 2011. Simulation of Brown-Resnick processes. Extremes. Accepted.

Rogers, L. C. G., Pitman, J. W., 1981. Markov functions. Ann. Probab. 9, 573-582.

Rogers, L. C. G., Williams, D., 2000. Diffusions, Markov processes, and martingales. Vol. 2. Cambridge Mathematical Library. Cambridge University Press, Cambridge.

Schlather, M., 2002. Models for stationary max-stable random fields. Extremes 5, 33-44.

Wang, Y., Stoev, S. A., 2010. On the structure and representations of max-stable processes. Adv. Appl. Probab. 42, 855-877. 